\title{
La contabilidad de costos en la producción de bienes y servicios. Revisión bibliográfica actualizada (2010-2018)
}

\author{
Cost accounting in the production of goods and services. \\ Updated Literature review (2010 -2018)
}

Recibido: 20-03-2018 • Aprobado:02-05-2018 • Página inicial: 203 - Página final: 230

\section{María Dolores Cabrera De Palacio*}

\begin{abstract}
Resumen: la producción de bienes y servicios es una actividad industrial de suma importancia en todas las sociedades. Constituye el motor que impulsa la economía global, no solo por el hecho de producir bienes y servicios, satisfactores de necesidades, sino también por todos los agentes que se ven involucrados en el proceso. Conociendo esto, el enfoque principal de esta investigación se dirige a la realización de una revisión bibliográfica actualizada, partiendo del año 2010, sobre los referentes bibliográficos que sirven de base para el estudio y análisis de los costos de producción en las carreras administrativas y financieras; ya que éstos deben registrarse, resumirse $\mathrm{y}$ analizarse para poder tomar decisiones empresariales. Para el estudio se utilizó el tipo de investigación documental, a partir de diferentes fuentes de datos: revisión en bibliotecas y librerías dominicanas, disponibilidades en casas editoras nacionales e internacionales; además del uso de herramientas digitales y motores de búsqueda en Internet.
\end{abstract}

Palabras clave: contabilidad, costos, producción, análisis, toma de decisiones.

\begin{abstract}
The manufacture of products and services is an industrial activity very important in all societies. It's the engine that drives the global economy, not only for the fact of manufacturing products and services satisfying needs, but also for all the agents that are involved in the process. Knowing this, the main focus of this research is directed to the accomplishment of an updated bibliographic revision, starting from the year 2010, on the bibliographical references that serve as base for the study and analysis of the costs of production in the administrative and financial careers, since they must be registered, summarized and analyzed in order to make business decisions. For the study, the type of documentary research was used, using different sources of data: review in Dominican libraries and bookstores, availability in national and international publishing houses, in addition to the use of digital tools and search engines on the Internet.
\end{abstract}

Keywords: Accounting, costs, production, analysis, decisions.

JEL: M41 - M49

\footnotetext{
* Contadora Pública y Magister en Contabilidad y Auditoría de la Universidad Nacional Pedro Henríquez Ureña (UNPHU). Investigadora y conferencista nacional e internacional. Docente certificada de contabilidad financiera, contabilidad de costos y sistemas de contabilidad en UNPHU.

mc6110@unphu.edu.do

Enlace ORCID: https://orcid.org/0000-0002-6836-2986
} 


\title{
Comptabilisation des coûts dans la production de biens et de services. Révision bibliographique mise à jour (2010 -2018)
}

\begin{abstract}
Résumé: la production de biens et de services est une activité industrielle de la plus haute importance dans toutes les sociétés. C'est le moteur de l'économie mondiale, non seulement pour le fait de produire des biens et des services, qui répondent à des besoins satisfaisants, mais également pour les agents impliqués dans le processus. Sachant cela, l'objectif principal de cette recherche est de réaliser une revue bibliographique actualisée, à partir de 2010, de références bibliographiques qui serviront de base à l'étude et à l'analyse des coûts de production des carrières administratives et financières; puisqu'ils doivent être enregistrés, résumés et analysés pour pouvoir prendre des décisions commerciales. Pour cette étude, le type de recherche documentaire a été utilisé, en fonction de différentes sources de données: examen des bibliothèques et des librairies dominicaines, disponibilité à la maison, chez des éditeurs nationaux et internationaux; outre l'utilisation d'outils numériques et de moteurs de recherche sur Internet.
\end{abstract}

Mots-clés: comptabilité, coûts, production, analyse, décisions.

\section{Contabilidade de custos na produção de bens e serviços. Revisão bibliográfica atualizada (2010 -2018)}

Resumo: a produção de bens e serviços é uma atividade industrial de extrema importância em todas as sociedades. É o motor que impulsiona a economia global, não apenas pelo fato de produzir bens e serviços, satisfazendo as necessidades, mas também pelos agentes envolvidos no processo. Sabendo disso, o foco principal desta pesquisa é direcionado para a conclusão de uma atualizado, a partir do ano de 2010, sobre as referências bibliográficas que são a base para o estudo e análise dos custos de produção nas carreiras administrativas e financeiras revisão da literatura; já que devem ser registrados, resumidos e analisados para tomar decisões de negócios. Para o estudo, utilizou-se o tipo de pesquisa documental, com base em diferentes fontes de dados: revisão de bibliotecas e livrarias dominicanas, disponibilidade em domicílio, editores nacionais e internacionais; além do uso de ferramentas digitais e motores de busca na internet.

Palarras-chave: contabilidade, custos, produção, análise, decisões. 


\section{Introducción}

Los temas que se tratan en esta investigación son: origen y evolución de la contabilidad de costos, la medición del costo de producción, registros contables, estados financieros y reportes de costos, análisis y toma de decisiones.

\section{Metodología}

Para realizar con éxito el estudio del tema se utilizó un tipo de investigación documental, revisando para ello: primero, programas académicos, nacionales e internacionales, que incluyen el estudio de la contabilidad de costos; segundo, fuentes bibliográficas en bibliotecas universitarias y librerías de la República Dominicana; tercero, disponibilidades en casas editoras, nacionales e internacionales; y cuarto, consulta en buscadores, bibliotecas y bases de datos digitales de uso internacional utilizados en la actualidad. ${ }^{1}$

La finalidad de estas revisiones es analizar la disponibilidad de referencias bibliográficas actualizadas en relación a las ofertas académicas y el contenido temático en materia de costos de producción.

\section{Resultados}

\section{La contabilidad de costos. Origen y evolución}

Diferentes autores, como Hernández (2012), Actualidad Empresarial (2014), Tafur y Osorio (2016), definen la contabilidad de costos como un subsistema contable que suministra información para medir los costos de un producto o servicio, calcular el rendimiento y establecer el control de las operaciones; siendo ésta indispensable en la gerencia de los negocios porque conoce con anterioridad a la producción, la inversión realizada y, por ende, le ayuda a tomar la decisión adecuada.

La contabilidad de costos se diferencia de la contabilidad financiera en que esta última recoge información de las operaciones económicas de la empresa, en sentido general, y elabora con ellas los estados financieros que pueden ser elaborados tanto para uso interno como externo. La de costos, "se basa en el registro y control de operaciones económicas por áreas, es elaborada para uso interno de la empresa y no es obligatoria" (Concepción, 2016; Hernández, 2012; Lazo, 2013a).

\footnotetext{
1 Algunos de los recursos digitales consultados fueron: Google académico, EBSCOhost, e-libro, freelibros.org, contabilidadparatodos.com, Youtube, entre otros.
} 


\section{Contexto cabrear, M.}

Su finalidad es suministrar información para: a) la valuación de los inventarios y la determinación de los resultados; b) el planeamiento y control de las operaciones de la empresa; y c) la toma de decisiones (Calleja, 2013; Jiménez, 2010; Lazo, 2013a; Ramírez, 2013).

En cuanto a sus orígenes, Hernández (2012) clasifica la historia de la contabilidad de costos basada en cuatro formas de producción: 1) Primitiva, caracterizaba por la producción solo de lo necesario para la subsistencia del grupo; 2) Esclavista, donde aparece y se desarrolla la propiedad privada sobre los medios de producción; 3) Feudal, ${ }^{2}$ donde los feudales eran dueños de los medios de producción (la tierra, molinos, animales, entre otros), los siervos solo eran dueños de sus instrumentos de labranza; y 4) Capitalista, caracterizada por la existencia de la propiedad privada sobre los medios de producción.En las cuatro formas de producción descritas, se han llevado crónicas de las operaciones en libros de registros, primero de forma rudimentaria, luego más especializada, a fin de mantener un control sobre ellas en los reinos, haciendas y comercios.

No obstante, varios autores, entre ellos Hernández (2012) y Lazo (2013a), afirman que el estallido de la Revolución Industrial, a partir del año 1776, fue lo que impulsó el establecimiento de un mayor control sobre los modos de producción, los materiales y la mano de obra. De esta industrialización surgieron cuatro tipos de actividades económicas: la comercialización, la prestación de servicios, la producción y la extracción o explotación.

Con respecto a lo anterior, Lazo (2013) especifica que fueron las industrias químicas las que hicieron evolucionar la contabilidad de costos.

En la actualidad, el aumento de la productividad, la maquinización y la tecnología industrial, han provocado el aceleramiento y la especialización de los modos de producción, lo que hace que el estudio de los procesos productivos y los costos inherentes a ellos se deban analizar con mucho cuidado.

Es por ello que hoy día las instituciones académicas incluyen la contabilidad de costos, administrativa, gerencial o de gestión en sus programas de estudio; dependiendo de la carrera de que se trate; contribuyendo así con los profesionales para que de manera competitiva identifiquen los costos en las empresas y puedan entender cómo clasificarlos, medirlos, registrarlos, analizarlos y presentarlos a la gerencia por medio de informes para la toma de decisiones.

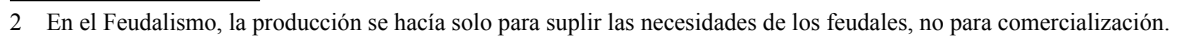


Además de lo anterior, tanto el contador de costos, el contador financiero o el administrador, deben saber que el trabajo con recursos materiales y económicos implica "cumplir con normas éticas que comprenden la competencia, integridad, confidencialidad y objetividad" (Horngren, Datar y Foster, 2012; Vanderbeck, 2017).

\section{Medición del costo de producción}

El costo es una erogación que se realiza para la adquisición de un bien o servicio con el propósito de generar ingresos (Jiménez, 2010; Warren, Reeve y Duchac, 2010).

Hernández (2012, 2014), Horngren et al. (2012b), Lazo (2013a), Tafur y Osorio (2013) y otros autores, identifican los costos como activo (inventarios y activos fijos), como gasto (costos de producción) y como pérdida (gasto). Lazo utiliza los términos costos capitalizables, inventariables y del período, para referirse a los mismos conceptos.

En cuanto a la composición y clasificación de los costos de producción, "la teoría tradicional de costos habla de tres elementos básicos: materiales directos, mano de obra directa y costos indirectos de fabricación" (Calleja, 2013; Hernández, 2012, 2014; Warren et al, 2010, 2012, 2014). A esta clasificación Rincón y Villarreal (2014a) y Tafur y Osorio (2013) agregan un cuarto elemento: el costo por contrato de servicios; también denominado como contratación de terceros, subcontratación, tercerización o externalización.

Estos elementos del costo, de manera general y para fines de medición, son clasificados como directos e indirectos (Ver Figura 1), si se relacionan con el producto; fijos, variables y mixtos, si se relacionan con el volumen producido; primos y de conversión, si se relacionan con los elementos del costo; de producción, administrativos, financieros o de ventas, de acuerdo al departamento en que se incurren; del producto y del período cuando se comparan con los ingresos (Díaz, 2010; Hernández, 2012, 2014; Horngren 2012a, 2012b; Lazo, 2013; Warren et al, 2010).

Los materiales, como madera, pegamentos, pinturas, harina, etc., "son los insumos que se transforman en productos terminados o servicios a través de la utilización de mano de obra y costos indirectos de fabricación en el proceso productivo" (Hernández, 2012, 2014; Lazo, 2013a; Warren et al, 2010). 


\section{Contexto caboba,,}

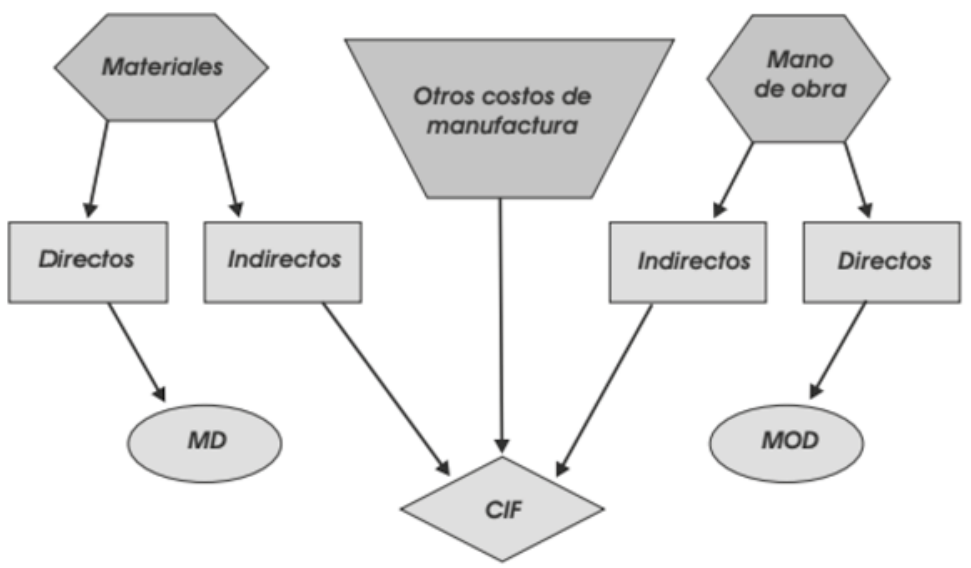

Figura 1. Elementos del costo de producción

Jiménez, 2010, p.18.

En su medición, es importante conocer los departamentos involucrados, las actividades de control, métodos de valoración, así como los documentos que deben elaborarse y que soportan la contabilidad (Ver flujograma de procesos presentado en la Figura 2) (Coyle, Langley, Novak y Gibson, 2013; Hernández, 2012; Heredia, 2013).

Además de lo anterior, Heredia (2013), Hernández (2012) y David (2016) recomiendan prestar atención a los términos comerciales utilizados en las compras internacionales, que implican trámites, acuerdos, cambios de moneda, conceptos referenciales, impuestos, gravámenes y permisos, transporte, seguros, almacenaje y manejo de documentos; todos ellos generadores de costos, los cuales deben contemplarse en liquidaciones de importación para establecer el costo final de adquisición de los materiales de producción. 

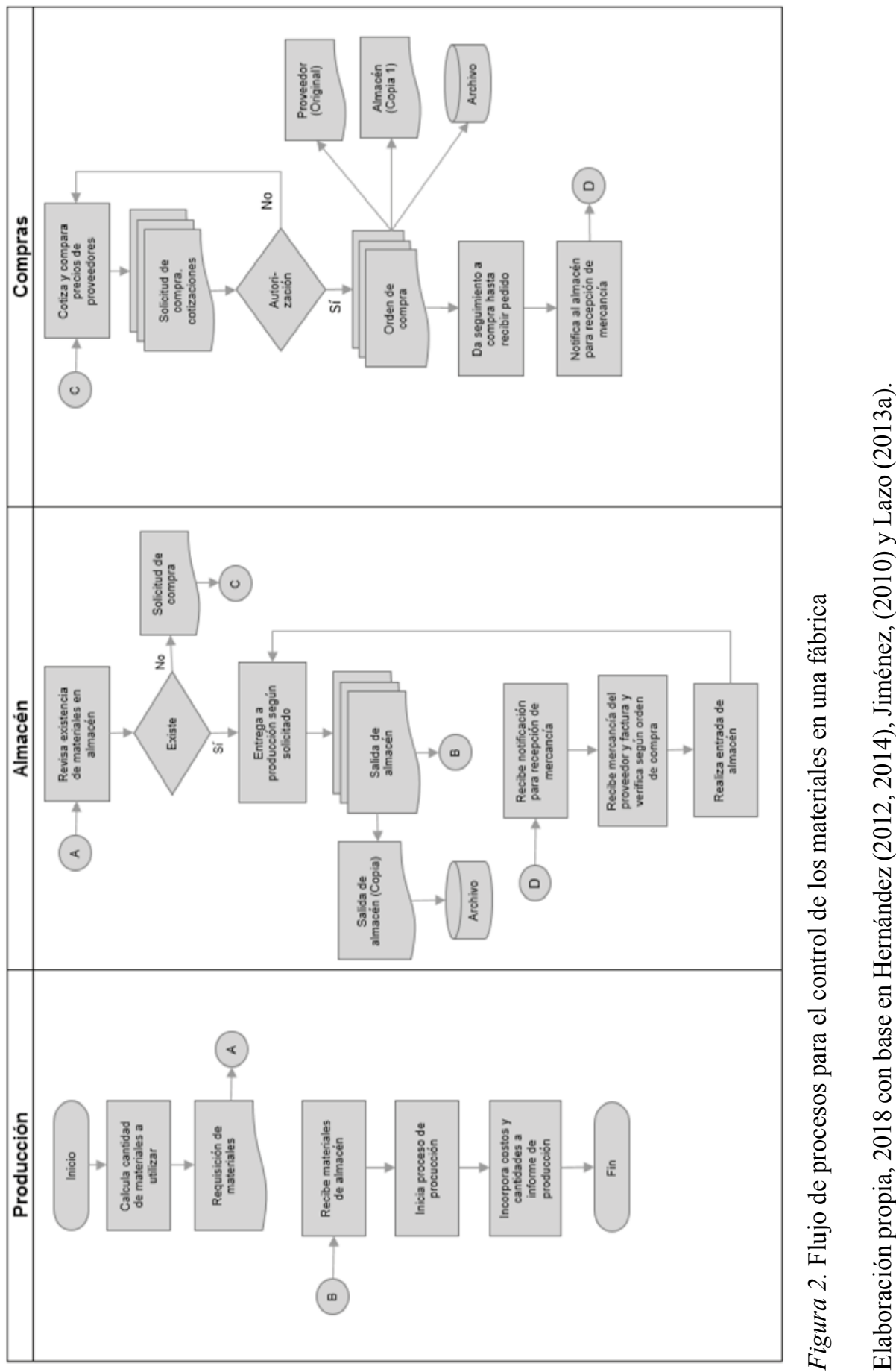


\section{Contexto caboras,,}

La mano de obra, el segundo elemento del costo, "es el esfuerzo físico o mental que realiza un trabajador en el proceso de transformación de una materia prima o un insumo en un producto terminado, que satisfaga una necesidad y que no perjudique el medio ambiente" (Hernández, 2012, 2014). Esta es administrada en la producción a través de la elaboración de boletas de trabajo, tarjetas de tiempo, nóminas de pago, y están regidas por el control de leyes y normativas de trabajo como son los códigos laborales, reglamentos sobre salarios mínimos, leyes sobre ISR y seguridad social, entre otros (Hernández, 2012; Lazo, 2013a).

No obstante, según Gómez (2013), el esfuerzo del ser humano sería insuficiente si no contara con los equipos necesarios que comprenden desde la herramienta más sencilla hasta la maquinaria más complicada, permitiendo economizar esfuerzos de trabajo y de material, a la vez que aumentar de modo significativo su productividad. Estos equipos sufren depreciación, se les realiza mantenimientos, utilizan energía o combustibles, los cuales deben considerarse como costos indirectos dentro de la producción.

Los costos indirectos de fabricación (CIF), carga fabril, gastos de fabricación o gastos, como también se les conoce (Jiménez, 2010), son todos los costos que se relacionan indirectamente con el producto y que forman parte del proceso productivo. Éstos se componen de tres partes: materiales indirectos, mano de obra indirecta y otros costos indirectos de fabricación (Ver Figura 1).

En procesos de construcción de obras, el costo indirecto parte desde la planificación de la edificación (costos preliminares), incluyendo los honorarios profesionales del personal a cargo y las subcontrataciones, hasta los costos finales de registro y evaluación de obras civiles (Suárez, 2017).

De los tres elementos básicos del costo, Donoso y Donoso (2011) aclaran que el CIF es el más difícil de calcular, por la variedad de costos y departamentos que se ven involucrados en ellos y las formas de distribución.

El cuarto elemento, subcontratación, aunque puede muy bien considerarse como CIF, Tafur y Osorio (2013) lo clasifican de tres formas: outsourcing (contratación de servicios externos realizados fuera de la empresa), cosoursing (realización de actividades compartidas con terceros, denominado también alianzas estratégicas) e insourcing (servicios externos realizados dentro de la empresa).

Los CIF generales pueden medirse y controlarse a través de la elaboración de presupuestos(Cano, 2013), hojas de prorrateo, hojas de consumoo documentaciones que soportan la carga del costo agregado a la producción (Hernández, 2014). 


\section{Registro de los costos de producción}

El registro de los costos de producción tiene dos objetivos: primero, elaborar informes que sirvan para la toma de decisiones y, segundo, presentar información "sobre el costo computable de los inventarios producidos" a la Administración Tributaria (SUNAT, DGII, Otras) (Abanto y Luján, 2013; Actualidad empresarial, 2014; Díaz, Parra y López, 2012).

Antes de contabilizar los costos de producción, debe conocerse primero cuáles tipos de inventarios se manejan y el sistema de costeo que se utilizará para su acumulación. Los inventarios en las industrias son tres: de materiales, de producción en proceso y de productos terminados (Hernández, 2014; Warren et al., 2010). En las empresas de servicios solo se consideran los inventarios de materiales (Vanderbeck, 2017).

En cambio, los sistemas de costeo de inventario son dos: periódico y perpetuo. El periódico, pormenorizado o de costos incompletos (Calleja, 2013), ofrece información limitada sobre los inventarios; se utiliza en pequeñas y algunas medianas empresas. El perpetuo o de costos completos, ofrece información en tiempo real y es utilizado en medianas y grandes empresas (Hernández, 2012, 2014; Lazo, 2013).

En las industrias, el sistema perpetuo permite la acumulación de costos de producción y lo clasifica de dos formas: por órdenes de trabajo y por procesos. El primero es en base a pedidos y especificaciones de clientes; sus costos se acumulan en una Hoja de Trabajo por cada pedido. El segundo es en base a procesos productivos continuos, estandarizados y repetitivos, donde los productos son homogéneos y producidos a gran escala (Horngren, 2012b; Uribe, 2011). Este último sistema acumula los costos por departamentos o por centros de costos.

Los centros de costos son unidades de trabajo que incluyen varias actividades en las cuales se van acumulando los tres elementos del costo, en donde los costos acumulados en un departamento pasan a otro para seguir el proceso, hasta que se termina la producción (Hernández, 2012, 2014; Lazo, 2013b).

Para los registros, los inventarios de materiales se contabilizan de dos formas: las entradas, al costo de adquisición (Ver primer asiento en Figura 3), principalmente contenido en las facturas de proveedores; las salidas se contabilizan de acuerdo al método de valoración de inventarios utilizado por la empresa. 


\section{Contexto caboba,,}

\begin{tabular}{|c|c|c|c|c|c|c|}
\hline & & Periódico & & Perpetuo & & \\
\hline \multirow[t]{2}{*}{$1 / 2 / x_{0}-$} & Compra & $\begin{array}{c}\text { Compras de materias } \\
\text { primas }\end{array}$ & 50 & Inventario de materiales & 50 & \\
\hline & & $\begin{array}{l}\text { Caja } \\
\text { (10 unidades } x \$ 5)\end{array}$ & 50 & Caja & & 50 \\
\hline \multirow[t]{2}{*}{$1 / 12 / \times 0 \cdots$} & $\begin{array}{l}\text { Materiales } \\
\text { enviados a } \\
\text { producción }\end{array}$ & No se hace asiento & & Trabajo en proceso & 20 & \\
\hline & & & & $\begin{array}{l}\text { Inventario de materiales } \\
\text { ( } 4 \text { unidades } x \$ 5 \text { ) }\end{array}$ & & 20 \\
\hline
\end{tabular}

Figura 3. Registro contable para compra de materiales

Lazo, 2013a, p.96.

Los métodos de valoración de inventarios conocidos son cuatro: FIFO (First In, First Out), LIFO (Last In, First Out), promedio ponderado y de identificación específica (Hernández, 2012; Lazo, 2013a). La NIC 2 solo hace mención de dos de ellos, FIFO e identificación específica, además del reconocimiento del valor neto realizable para contabilizar (Díaz, 2010; Instituto Mexicano de Contadores Públicos, 2016).

De los métodos citados, en las industrias solo se utilizan el FIFO y el Promedio ponderado (Donoso y Donoso, 2011; Jiménez, 2010), por el tipo de procesos y el momento en que se agregan los costos de los inventarios; pero cada país, a través de la administración tributaria, determinará cuál es el más favorable a utilizar. Por ejemplo, en la República Dominicana la Dirección General de Impuestos Internos (DGII) exige en el Código Tributario, Ley 11-92, utilizar LIFO, salvo ciertas excepciones (Hernández, 2014). En Perú, la forma de registro de costos está contenida en la "ley del impuesto a la renta" a partir del artículo 35, sobre inventarios y contabilidad de costos (Aguirre, 2013).

El registro de los materiales se debe realizar considerando "la cantidad recibida, cantidad enviada a producción, y los saldos de los inventarios para cada tipo de material". A medida que se usan materiales directos, se registran como materiales retirados del almacén en el mayor de fábrica (Horngren, 2012b). En el segundo asiento de la Figura 3, las salidas del almacén de materiales se computan directamente a la cuenta de inventario de producción en proceso, cuando se utiliza el sistema perpetuo, cuenta donde se acumulan los costos del producto a fabricar. En el sistema periódico no se realiza asiento contable para el traspaso de materiales (Lazo, 2013a), por lo que el control debe hacerse manual en las hojas de trabajo. 
Con relación al registro de la mano de obra debe definirse si el costo se cargará directamente a la producción o a una cuenta de control y acumulación de costos desde la cual será distribuido. Esto sucede cuando existen varias órdenes de trabajo, departamentos o centros de costos trabajándose al mismo tiempo (Ver Figura 4). Jiménez (2010), indica que los aportes sociales a los empleados también deben incluirse en la cuenta de producción en proceso de cada departamento u orden de trabajo.

\begin{tabular}{|lll|}
\hline Trabajo en proceso --- Departamento A & 5000 & \\
Trabajo en proceso --- Departamento B & 6200 & \\
Trabajo en proceso --- Departamento C & 4800 & \\
Nómina & & 16000 \\
\hline
\end{tabular}

Figura 4. Registro contable para la mano de obra directa

Lazo, 2013b.

En caso de utilizarse un registro por orden de trabajo, en vez de clasificar los registros por departamentos se asignan a trabajo en proceso de cada orden.

Para el registro de los CIF, debe considerarse que éstos se agregan a la producción utilizando diferentes bases de cálculo: a) costo de materiales directos; b) costo de mano de obra directa; c) horas de mano de obra directa; d) unidades producidas; y e) horas máquina; (Hernández 2012, 2014; Lazo, 2013a). También se pueden asignar mediante el uso del sistema de costeo ABC. (Concepción, 2016; Pabón, 2012; Ramírez, 2013).

La Figura 5 muestra cómo debe realizarse el registro de este elemento en la producción, tomando en cuenta que previamente se fueron acumulando en una cuenta de control de CIF, la cual finalmente es distribuida entre los departamentos u órdenes de producción. En caso de computarse directamente a la producción, sin pasar por la cuenta de acumulación, la partida crédito será la cuenta de obligación o salida de efectivo correspondiente.

Cada costo en la producción puede agregarse en base a costos reales, normales o estándar. En algunos casos esta asignación se basa en tasas predeterminadas, siendo acumulados en una cuenta de acumulación y luego distribuidas por tasas o de acuerdo a la proporción producida. Estas formas de control servirán para tomar decisiones, sea utilizando un enfoque de costeo directo o por absorción. 


\section{Contexto caboras,$M$}

$\begin{array}{ll}\text { Trabajo en proceso -.-.-. Departamento A } & 7500 \\ \text { Trabajo en proceso -.-.- Departamento B } & 9300 \\ \text { Trabajo en proceso -.-.- Departamento C } & 7200\end{array}$

Control de costos indirectos de fabricación

Figura 5. Contabilización de los costos indirectos de fabricación

Lazo, 2013b.

No se debe confundir el sistema de costeo ABC con la distribución de los costos de producción entre los departamentos $\mathrm{A}, \mathrm{B}$ y C.

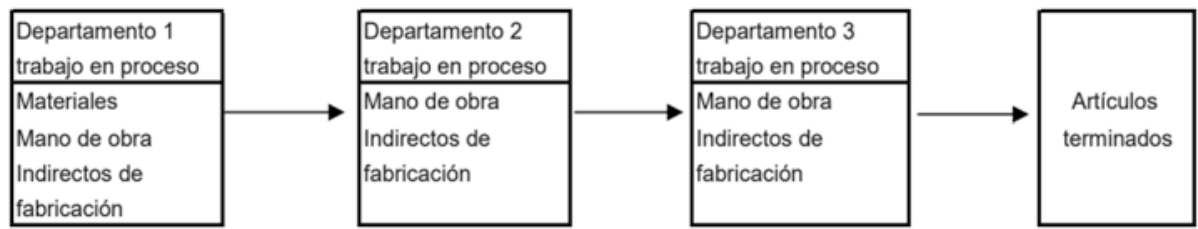

Figura 6. Flujo secuencial en la producción

Lazo, 2013b, p.52.

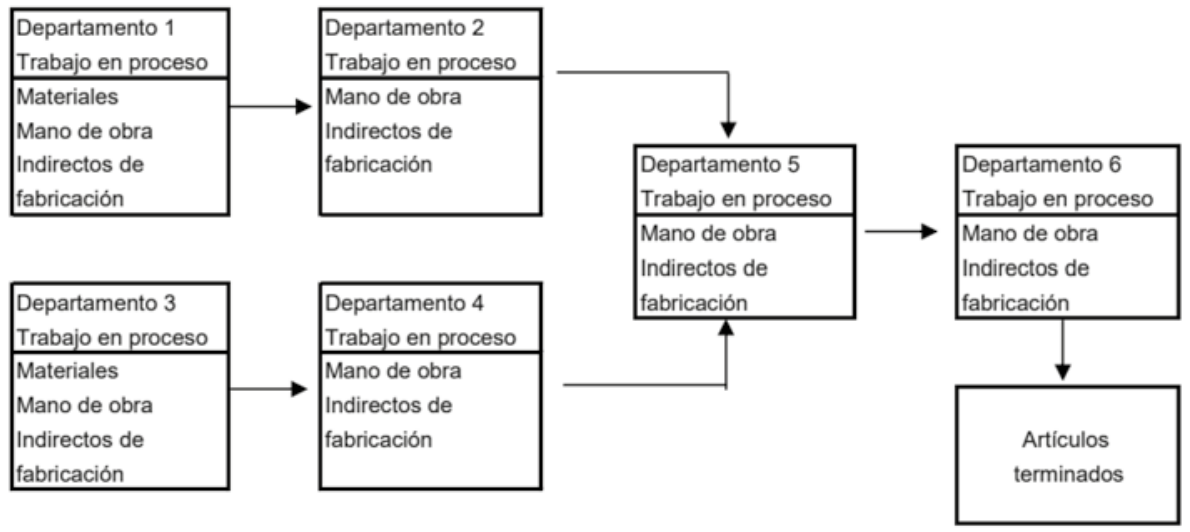

Figura 7. Flujo paralelo en la producción

Lazo, 2013b, p.52. 


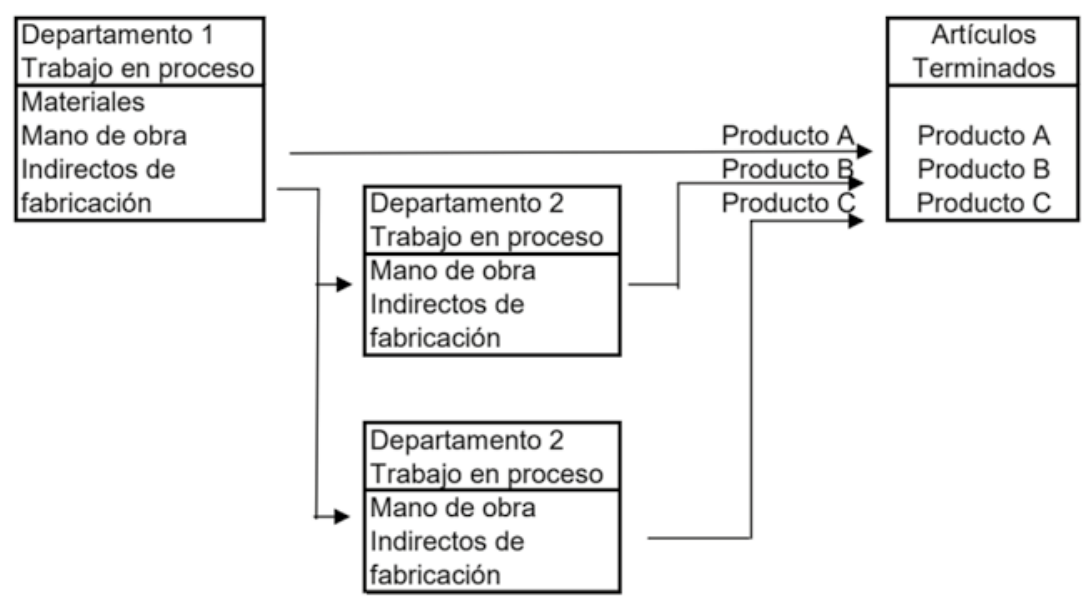

Figura 8. Flujo paralelo en la producción

Lazo, 2013b, p.53.

Independientemente del sistema de registro que se utilice, periódico o perpetuo, o del sistema de acumulación de costos, por órdenes de trabajo o por procesos, Lazo (2013b), señala que es importante saber que el proceso productivo podrá ser secuencial, paralelo o selectivo; dependiendo de la forma en que los productos y los costos pasan por los diferentes departamentos. Para entender bien esto se muestran las Figuras 6,7 y 8 .

Así mismo, algunos procesos productivos se realizan de manera conjunta, en donde productos diferentes pueden resultar de un mismo proceso inicial en el primer o los primeros departamentos (Ver Figura 9) (Cabrera y Mariano, 2015; Concepción, 2016).

La NIC 2 define producción conjunta como: el proceso de producción que puede dar lugar a la fabricación simultánea de más de un producto (Párrafo 14); resultando de ello la producción de productos principales o coproductos (productos de valor de venta significativo) junto a subproductos (desechos, desperdicios o rechazos) (Cabrera y Mariano, 2015).

Como se puede notar en la Figura 9, no se pueden identificar los productos que se tiene intención de fabricar, $\mathrm{A}, \mathrm{B}$ y $\mathrm{C}$, sino hasta después del punto de separación (en donde pueden identificarse individualmente); por lo que, en el primer departamento, solamente se pueden identificar los costos que se acumulan para los tres productos de forma simultánea (costos conjuntos) (Cabrera y Mariano, 2015). 


\section{Contexto caboras,,}

La contabilización en la producción conjunta se realiza de la misma forma que se ha presentado en las Figuras 3, 4 y 5. Cuando se utilizan varios departamentos las cuentas de inventario de producción en proceso de cada departamento estará identificada con la letra, código o nombre del departamento correspondiente, para identificar con mayor facilidad dónde se ha generado o traspasado el costo incurrido.

Para asignar los costos conjuntos a los coproductos, Cabrera y Mariano (2015) y Concepción (2016) señalan que se deberá tomar en cuenta el método de asignación de costos a utilizar; ya sea tomando en cuenta las unidades físicas (método de las unidades producidas) o su importancia en los ingresos (métodos de valor de venta en el punto de separación y del valor neto realizable).

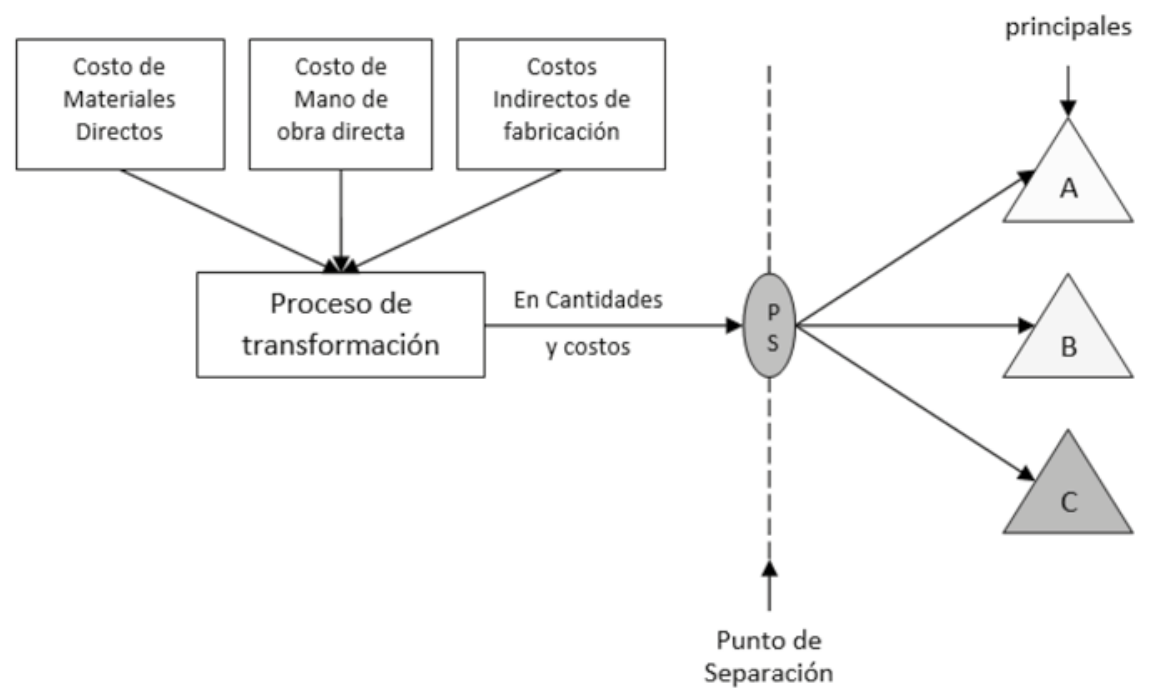

Figura 9. Flujo de costos en la producción conjunta

Cabrera y Mariano, 2015.

El primer método, de las unidades producidas, se utiliza cuando el valor de venta de los coproductos en el mercado no tienen diferencia significativa en los ingresos de la empresa; por lo que, la cantidad producida será la base para la asignación de costos. La siguiente fórmula se utiliza para ello (Cabrera y Mariano, 2015). 


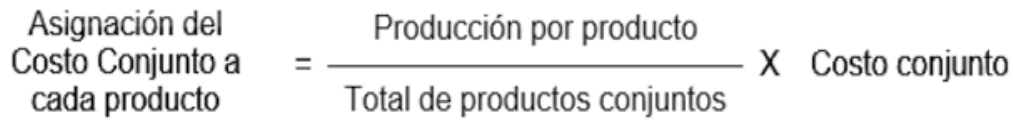

El segundo método, del valor de venta en el punto de separación, es el más idóneo en la producción conjunta, pero solo cuando se conoce el valor de venta unitario de los productos en este punto. Estos son los productos que son vendidos inmediatamente; se identifica individualmente que no requieren de procesamiento adicional y casi siempre se venden a granel. Este método se utiliza mucho en las procesadoras de carnes. La fórmula a utilizar en este caso, presentada por cabrera y Mariano (2015), es la siguiente:

\begin{tabular}{|c|c|c|c|}
\hline Asignación del & & $\begin{array}{l}\text { Valor total de venta en el punto de } \\
\text { separación de cada producto* }\end{array}$ & Costo \\
\hline $\begin{array}{l}\text { Costo Conjunto a } \\
\text { cada producto }\end{array}$ & $=$ & $\begin{array}{l}\text { Valor total de venta en el punto de } \\
\text { separación de todos los productos }{ }^{+}\end{array}$ & conjunto \\
\hline $\begin{array}{l}\text { "Valor total de venta de } \\
\text { cada producto }\end{array}$ & $=$ & \multicolumn{2}{|c|}{$\begin{array}{l}\text { Unidades producidas de cada producto } \times \text { Valor unitario de venta en el punto de } \\
\text { separación de cada producto }\end{array}$} \\
\hline $\begin{array}{l}\text { "Valor total de venta de } \\
\text { todos los productos }\end{array}$ & $=$ & \multicolumn{2}{|c|}{ Suma de los valores de venta de todos los productos individuales } \\
\hline
\end{tabular}

El tercer método, del valor neto realizable, se utiliza cuando no se conoce el valor de venta en el punto de separación. Para ello, debe utilizarse el precio de venta final del producto, considerando reducir de éste los costos de procesamiento adicional y los gastos operacionales cargados a los productos. De esta forma, se obtiene un valor hipotético de venta en el punto de separación. La forma de obtener este valor hipotético, Cabrera y Mariano (2015) lo presentan en la siguiente fórmula:

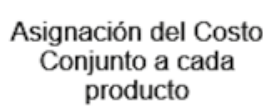
"Valor total hipotético de
venta de cada producto

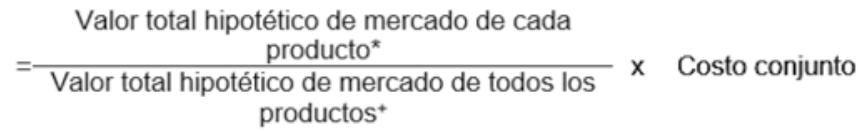

- (Unidades producidas de cada producto $\mathrm{x}$ Valor unitario de venta final de cada producto)
= Costos de procesamiento adicional y gastos de venta de cada producto

\footnotetext{
= Suma de los valores hipotéticos de venta de todos los productos individuales

•Valor total hipotético de venta de todos los productos

Sule de los valores hipotéticos de venta de todos los productos individuales
} 


\section{Contexto caboras,,}

Para poner un ejemplo sobre los cálculos realizados por cada uno de los métodos de asignación de costos se presentan las Figuras 10, 11 y 12, correspondientes a las Tablas I, II y III expuestas por Cabrera y Mariano en su tesis de maestría (2015), en base a una industria procesadora de carnes de pollo.

\begin{tabular}{|c|c|c|c|c|c|c|c|}
\hline & A & B & \multirow{2}{*}{\multicolumn{2}{|c|}{ Proporción }} & D & \multirow{2}{*}{\multicolumn{2}{|c|}{$\begin{array}{c}E \\
\begin{array}{c}\text { Asignación de Costo } \\
\text { Conjunto }\end{array}\end{array}$}} \\
\hline 1 & Producto & $\begin{array}{l}\text { Cantidad } \\
\text { Producida }\end{array}$ & & & $\begin{array}{l}\text { Costo } \\
\text { Conjunto }\end{array}$ & & \\
\hline 2 & A. Muslos con costillas & 142.20 & $\mathrm{~B} 2 / \mathrm{B} 5=$ & 0.49000689 & $\operatorname{RD} \$ 13,340.80$ & $\mathrm{C} 2 \times \mathrm{D} 2=$ & RD\$ $6,537.08$ \\
\hline 3 & B. Alas especiales & 36.10 & $\mathrm{~B} 3 / \mathrm{B} 5=$ & 0.12439697 & RD\$13,340.80 & $\mathrm{C} 3 \times \mathrm{D} 3=$ & RD\$ $1,659.56$ \\
\hline 4 & C. Pechugas con hueso & 111.90 & $\mathrm{~B} 4 / \mathrm{B} 5=$ & 0.38559614 & RD\$13,340.80 & $\mathrm{C} 4 \times \mathrm{D} 4=$ & RD\$ 5,144.16 \\
\hline 5 & Subtotal & 290.20 & & 1.00000000 & & & RD\$13,340.80 \\
\hline 6 & D. Huesos & 13.00 & & & & & \\
\hline 7 & Total & 303.20 & & & & & \\
\hline
\end{tabular}

Figura 10. Ejemplo en base al método de las unidades producidas

Cabrera y Mariano, 2015, p.34.

Como puede percibirse, en cada uno de los métodos, la distribución de los costos conjuntos es diferente, porque el peso de la cantidad producida y los precios de venta indicarán hacia dónde debe ir mayor carga de costos, por lo tanto, la gerencia debe determinar qué método es el más factible usar.

\begin{tabular}{|c|c|c|c|c|c|c|c|c|c|c|}
\hline & A & B & C & \multirow{2}{*}{\multicolumn{2}{|c|}{$\begin{array}{l}\text { Precio de venta total en el } \\
\text { punto de separación }\end{array}$}} & & $E$ & $F$ & & $G$ \\
\hline 1 & Producto & $\begin{array}{l}\text { Cantidad } \\
\text { Producida }\end{array}$ & $\begin{array}{l}\text { Precio de } \\
\text { venta } \\
\text { unitario en } \\
\text { el punto de } \\
\text { separación }\end{array}$ & & & \multicolumn{2}{|c|}{ Proporción } & $\begin{array}{l}\text { Costo } \\
\text { Conjunto }\end{array}$ & \multicolumn{2}{|c|}{$\begin{array}{c}\text { Asignación de Costo } \\
\text { Conjunto }\end{array}$} \\
\hline 2 & Muslos con costillas & 142.20 & RDS 52.00 & $\mathrm{~B} 2 \times \mathrm{C} 2=$ & RDS $7,394.40$ & $\mathrm{D} 2 / \mathrm{D} 5=$ & 0.40415833 & RD $\$ 13,340.80$ & $E 2 \times F 2=$ & RDS $5,391.80$ \\
\hline 3 & Alas especiales & 36.10 & RDS 54.00 & $\mathrm{~B} 3 \times \mathrm{C} 3=$ & RDS $1,949.40$ & $\mathrm{D} 3 / \mathrm{D} 5=$ & 0.10654904 & $\operatorname{RD} \$ 13,340.80$ & $E 3 \times F 3=$ & $\operatorname{RDS} 1,421.45$ \\
\hline 4 & Pechugas con hueso & 111.90 & RDS 80.00 & $\mathrm{~B} 4 \times \mathrm{C} 4=$ & RDS 8.952 .00 & $\mathrm{D} 4 / \mathrm{D} 5=$ & 0.48929262 & RDS13,340.80 & $\mathrm{E} 4 \times \mathrm{F} 4=$ & RDS 6.527 .56 \\
\hline 5 & Subtotal & 290.20 & & & RD\$18,295.80 & & 1.00000000 & & & $\operatorname{RD} \$ 13,340.80$ \\
\hline 6 & Huesos & 13.00 & & & & & & & & \\
\hline 8 & Total & 303.20 & & & & & & & & \\
\hline
\end{tabular}

Figura 11. Ejemplo en base al método del valor de venta en el punto de separación

Cabrera y Mariano, 2015, p.36. 


\begin{tabular}{|c|c|c|c|c|c|c|c|c|c|}
\hline \multirow[b]{2}{*}{1} & \multirow[b]{2}{*}{ Producto } & \multirow[b]{2}{*}{$\begin{array}{c}\text { Cantidad } \\
\text { Producida* }\end{array}$} & \multirow{2}{*}{$\begin{array}{c}\text { C } \\
\text { Precio de } \\
\text { venta } \\
\text { unitario } \\
\text { final }\end{array}$} & \multicolumn{2}{|r|}{ D } & \multicolumn{2}{|r|}{$E$} & \multicolumn{2}{|r|}{$\mathrm{F}$} \\
\hline & & & & \multicolumn{2}{|c|}{ Precio de venta total final } & \multicolumn{2}{|c|}{$\begin{array}{l}\text { Procesam. } \\
\text { adicional }\end{array}$} & \multicolumn{2}{|c|}{$\begin{array}{l}\text { Valor hipotético de venta } \\
\text { en el punto de separación }\end{array}$} \\
\hline 2 & Muslos con costillas & 190.00 & RD\$ 40.00 & $\mathrm{~B} 2 \times \mathrm{C} 2=$ & RDS $7,600.00$ & RDS & $2,584.00$ & $\mathrm{D} 2-\mathrm{E} 2=$ & RDS $5,016.00$ \\
\hline 3 & Alas especiales & 36.10 & RD\$50.00 & $\mathrm{B} 3 \times \mathrm{C} 3=$ & RDS $1,805.00$ & RDS & 54.15 & $\mathrm{D} 3-\mathrm{E} 3=$ & RDS $1,750.85$ \\
\hline 4 & Pechugas con hueso & 142.80 & RD $\$ 90.00$ & $\mathrm{~B} 4 \times \mathrm{C} 4=$ & $\operatorname{RD} \$ 12,852.00$ & RDS & $3,598.56$ & $\mathrm{D} 4-\mathrm{E} 4=$ & RDS $9,253.44$ \\
\hline 5 & Subtotal & 368.90 & & & RD\$22,257.00 & RDS & $6,236.71$ & & RD\$16,020.29 \\
\hline 6 & Huesos & 13.00 & & & & & & & \\
\hline 7 & Total & 381.90 & & & & & & & \\
\hline
\end{tabular}

\begin{tabular}{|c|c|c|c|c|c|c|c|}
\hline & A & $\mathrm{F}$ & & G & $\mathrm{H}$ & & 1 \\
\hline 1 & Producto & $\begin{array}{c}\text { Valor hipotético } \\
\text { de venta en el } \\
\text { punto de } \\
\text { separación }\end{array}$ & \multicolumn{2}{|c|}{ Proporción } & $\begin{array}{l}\text { Costo } \\
\text { Conjunto }\end{array}$ & \multicolumn{2}{|c|}{$\begin{array}{c}\text { Asignación de Costo } \\
\text { Conjunto }\end{array}$} \\
\hline 2 & \multirow{5}{*}{$\begin{array}{l}\text { Muslos con costillas } \\
\text { Alas especiales } \\
\text { Pechugas con hueso } \\
\text { Subtotal } \\
\text { Huesos } \\
\text { Total }\end{array}$} & RDS $5,016.00$ & \multirow{5}{*}{$\begin{array}{l}\mathrm{F} 2 / \mathrm{F} 5= \\
\mathrm{F} 3 / \mathrm{F} 5= \\
\mathrm{F} 4 / \mathrm{F} 5=\end{array}$} & 0.31310295 & \multirow{5}{*}{$\begin{array}{l}\text { RD } \$ 13,340.80 \\
\text { RD\$13,340.80 } \\
\text { RD\$13,340.80 }\end{array}$} & \multirow{5}{*}{$\begin{array}{l}\mathrm{G} 2 \times \mathrm{H}_{2}= \\
\mathrm{G} 3 \times \mathrm{H} 3= \\
\mathrm{G} 4 \times \mathrm{H} 4=\end{array}$} & RD\$ $4,177.04$ \\
\hline 3 & & RDS 1.750 .85 & & 0.10928953 & & & RD\$ $1,458.01$ \\
\hline 4 & & RDS $9,253.44$ & & 0.57760752 & & & RD\$ $7,705.75$ \\
\hline 5 & & RD\$16,020.29 & & 1.00000000 & & & RD $\$ 13,340.80$ \\
\hline $\begin{array}{l}6 \\
7\end{array}$ & & & & & & & \\
\hline
\end{tabular}

Figura 12. Ejemplo en base al método del valor neto realizable

Cabrera y Mariano, 2015, p.39.

Los subproductos en las Figuras 10, 11 y 12 no aplican para la asignación de costos de producción, porque se consideran como "subproductos de categoría 1, los cuales solo se reconocen cuando se venden". Los subproductos de categoría 2, una segunda clasificación, se someten al mismo proceso de asignación de costos que los coproductos, "por reconocerse cuando se producen"; por tanto, deben ser cargados con costos del proceso productivo (Cabrera y Mariano, 2015).

Después de obtener la asignación de costos en el punto de separación, se procede al registro del traspaso a los siguientes departamentos o al almacén de productos terminados, dependiendo si se realizará o no un proceso adicional de producción (ver segundo registro en Figura 13).

Finalmente, terminada la producción se realizan los registros correspondientes al traspaso de los productos terminados al almacén, en donde se les asigna un precio de venta con el cual se comercializará al cliente. En la Figura 14, Horngren et al. (2012b), muestran los registros de traspaso utilizando una cuenta de "control de productos terminados"; la misma utilizada por otros autores para el inventario de productos terminados. La Figura también presenta los asientos contables a realizar al momento de vender los artículos. 


\section{Contexto caboras,,}

Comercial La Gran Parada, S.R.L.

Diario General

\begin{tabular}{|c|c|c|c|}
\hline Fecha & Detalle & Débito & Crédito \\
\hline \multirow[t]{6}{*}{$x x-x x-x x x x$} & Inventario de producción en proceso - Corte & $13,340.80$ & \\
\hline & @ & & \\
\hline & Inventario de materia prima & & $10,612.00$ \\
\hline & Nómina por pagar & & $2,046.60$ \\
\hline & Costos indirectos de fabricación aplicados & & 682.20 \\
\hline & $\begin{array}{l}\text { Registro costos agregados a la producción, } \\
\text { sección de corte. }\end{array}$ & & \\
\hline \multirow[t]{6}{*}{$x x-x x-x x x x$} & Inventario de producción en proceso - Inyección & $5,391.80$ & \\
\hline & Inventario de producción en proceso - Empaque & $1,421.45$ & \\
\hline & Inventario de producción en proceso - Deshuese & $6,527.56$ & \\
\hline & (a) & & \\
\hline & Inventario de producción en proceso - Corte & & $13,340.80$ \\
\hline & $\begin{array}{l}\text { Registro entrega producción de Corte a } \\
\text { Inyección, Deshuese y Empaque. }\end{array}$ & & \\
\hline
\end{tabular}

Figura 13. Registros contables en producción conjunta

Cabrera y Mariano, 2015, p.41.

6. Terminación y transferencia de las órdenes de trabajo individuales a productos terminados, $\$ 188,800$.

Control de productos terminados Control de productos en proceso

188,800

188,800

7. Costo de ventas, $\$ 180,000$.

Costo de ventas

180,000

Control de productos terminados

180,000

8. Costos de marketing de febrero, $\$ 45,000$, y costos de los servicios al cliente de febrero, $\$ 15,000$, pagados en efectivo.
Gastos de marketing
45,000
Gastos de servicio al cliente
15,000
Control de efectivo
60,000

9. Ingresos por ventas, todos ellos a crédito, $\$ 270,000$.

Control de cuentas por cobrar Ingresos

270,000

270,000

Figura 14. Contabilización de la producción transferida a productos terminados

Horngren et al. (2012b, p.114). 
Cabe resaltar que, en todo proceso productivo pueden resultar materiales de desecho y de desperdicio y unidades dañadas y defectuosas que deben considerarse a la hora de calcular y registrar costos (Warren et al, 2010).

Los desperdicios o daños en la producción pueden ser normales o anormales. Los primeros impactan los costos de producción, y por tanto la producción terminada y el costo de ventas, porque son absorbidos en el costo de la producción buena; en cambio, los desperdicios y daños anormales, se cargan a los deudores, a la cuenta del responsable, o bien a la empresa, en forma de gastos (Avanto y Luján, 2013; Cabrera y Mariano, 2015; Calleja, 2013; Uribe, 2011).

Para evitar esos daños y desperfectos en la producción, se incurre en procesos y costos de calidad que también deben ir cargados a la producción dentro de los costos indirectos. También existen costos de mala calidad (de prevención y evaluación), los cuales se producen por la detección de fallas internas (antes de los productos ser entregados a los clientes) o fallas externas (surgidos una vez han sido entregados) encontradas en los productos (Lazo 2013b; Uribe, 2011; Vanderbeck 2017).

\section{Informes de costos y estados financieros}

Cuando se termina una orden o proceso productivo, se da aviso al departamento de costos para que proceda a la liquidación de los costos incurridos a través de informes de producción. Jiménez (2010) especifica que esta se efectúa totalizando los costos de materiales directos, mano de obra directa y CIF en la respectiva Hoja de Costos, para luego dividirlo entre el número de unidades producidas y alcanzar así el costo promedio por unidad.

En la producción por procesos se elabora un informe de costo de producción por departamentos que indicará cuál fue el costo total producido, la cantidad de unidades terminadas y transferidas, unidades en proceso y mermas, y el costo unitario resultante. Este informe algunos autores, como Hernández (2012, 2014) y Warren et al. (2010), lo describen en cuatro pasos: plan de cantidades, producción equivalente, costos por contabilizar y costos contabilizados, pudiéndose agrupar en dos partes: unidades, pasos 1 y 2 , y costos, pasos 3 y 4 . Otros autores, sin embargo, como Horngren (2012a, 2012b) dividen el mismo informe en cinco partes: cantidades, producción equivalente, costos totales invertidos, costos unitarios equivalentes y costos asignados. 
Cuando los costos se contabilizan y resumen, sirven para la elaboración de los estados financieros básicos (balance general, estado de resultados, estado de flujo de efectivo, estado de cambios en el patrimonio), auxiliándose de la contabilidad de costos a través de la elaboración de los estados financieros complementarios (estado de costos de producción y de costos de la mercancía vendida) (García, 2014; Hernández, 2012, 2014).

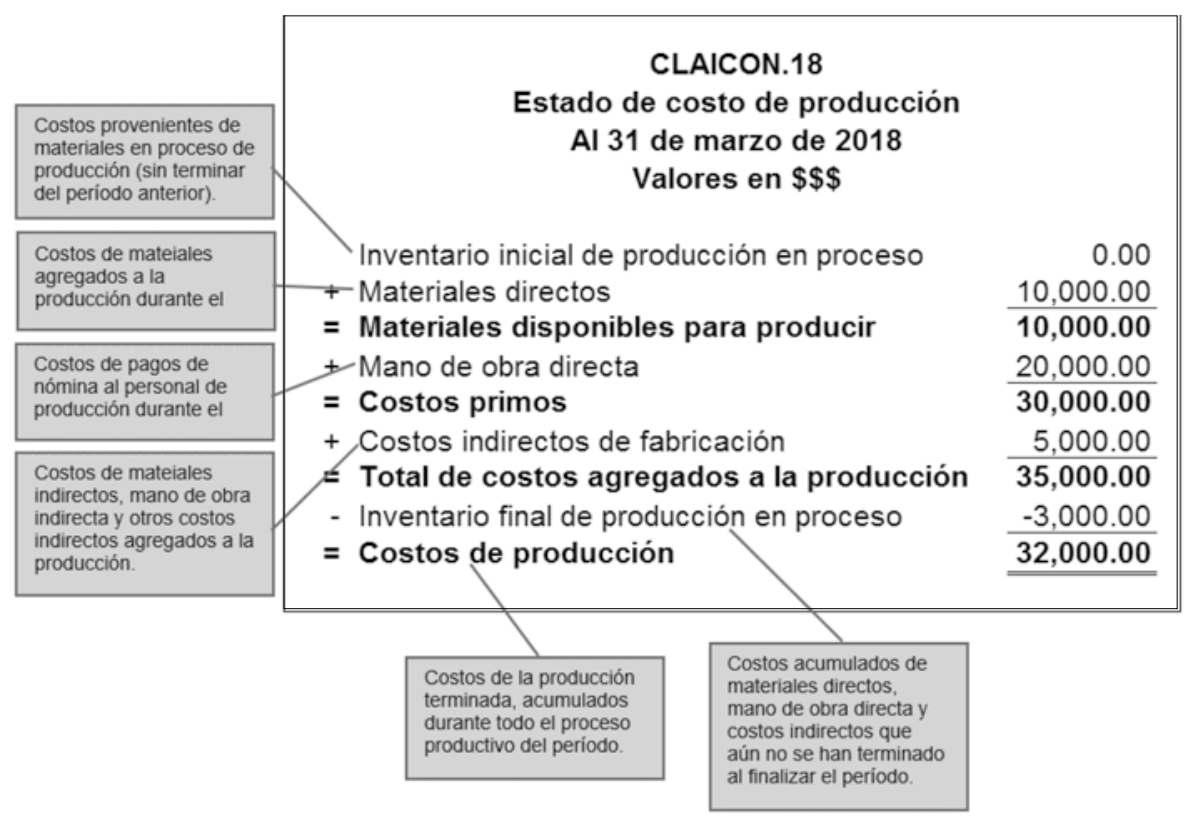

Figura 15. Modelo de estado de costo de producción

Elaboración propia, 2018 con base en descripciones de Cano (2013), Hernández (2012) y Warren et al (2010).

Las Figuras 15 y 16 muestran dos modelos de estados financieros secundarios, indicando en ellos la ubicación de los costos y de dónde son obtenidos. El resultado del estado de costo de producción (Figura 15), sirve para la elaboración del estado de costo de ventas (Figura 16), cuyo resultado se utiliza para la elaboración del estado de resultado (disminuido de los ingresos), el cual servirá para alimentar los demás estados financieros básicos. "Estos estados secundarios pueden prepararse aun cuando la empresa no cuente con contabilidad de costos", siendo el estado de costo de producción tan complejo como lo sea el proceso productivo (Calleja, 2013; Lazo, 2013a). 
En una empresa de prestación de servicios no existe costo de mercancía vendida. Se calcula el costo de los servicios prestados, el cual está compuesto de costo de materiales directos, costo de mano de obra directa, costos indirectos de la prestación del servicio y costo de subcontrataciones (Uribe 2011; Vanderbeck, 2017).

Los estados financieros básicos se elaboran en las industrias y empresas de servicios de igual forma como se conoce en las empresas comerciales.

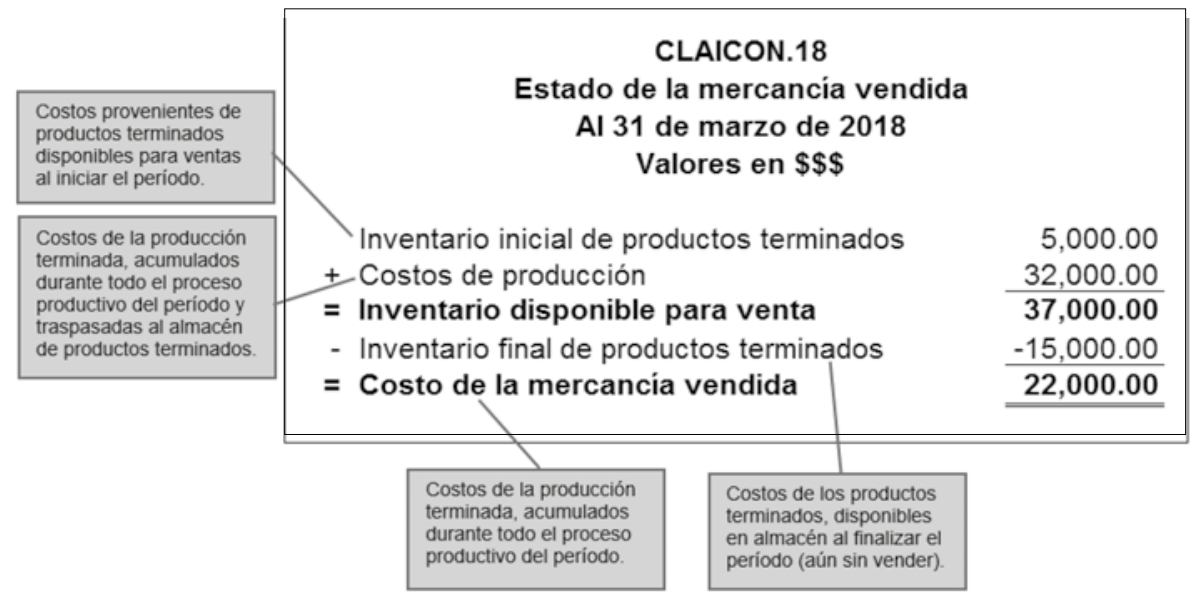

Figura 16. Modelo de estado de costo de la mercancía vendida

Elaboración propia, 2018 con base en descripciones de Cano (2013), Hernández (2012) y Warren et al (2010).

\section{Análisis de los costos de producción}

En las finanzas tradicionales, existen indicadores de rentabilidad, liquidez y endeudamiento. Algunos indicadores de rentabilidad importantes son: margen bruto (utilidad bruta/ventas netas), margen operacional (utilidad operacional/ ventas netas) y margen neto (utilidad neta/ventas brutas). Estos tres indicadores miden la capacidad que tienen las ventas de generar utilidades (Uribe, 2011).

En las industrias, aparte de los indicadores tradicionales, también existen los indicadores de rotación de inventarios, calculados para cada tipo de inventario utilizado en estos tipos de empresas, de productividad y competitividad de eficiencia y de calidad (Hernández, 2012; Uribe, 2011). 


\section{Contexto cabrear, M.}

La finalidad del análisis permitirá evaluar los costos desde diferentes enfoques. Por ello, Hernández (2012), Horngren et al. (2012a, 2012b), Warren et al. (2010) y otros autores los clasifican como: históricos y predeterminados, estimados, normales y estándares, directos y absorbentes, controlables e incontrolables, relevantes e irrelevantes, desembolsables y de oportunidad, sumergidos y diferenciales, evitables e inevitables, comprometidos y discrecionales, de cierre de planta, entre otras clasificaciones.

En base a la clasificación anterior, Suárez (2017) expone que los análisis pueden ser aproximados, específicos, dinámicos, deductivos o inductivos. También describe que el costo está precedido de costos anteriores y que, a su vez, son integrantes de costos posteriores.

Los costos históricos se refieren a los costos reales, los cuales deben compararse, cuando se efectúan, con costos predeterminados (estimados y estándares), a fin de evaluar la flexibilidad presupuestaria, analizando las variaciones resultantes en la aplicación de costos. Estas variaciones pueden deberse a subaplicaciones, sobreaplicaciones o a cambios en los precios y regulaciones del mercado después de aprobadas las partidas presupuestarias (Hernández, 2012; Horngren et al 2012a, 2012b).

El proceso presupuestal se inicia con la determinación del nivel de ventas, porque dependiendo de estas se establecen las necesidades de materiales directos e indirectos, mano de obra, costos indirectos de fabricación, inversión en activos fijos, necesidades de financiación o de inversión, niveles de inventarios, racionalización de gastos y programación de pagos. Al conjunto de estos presupuestos se le denomina presupuesto maestro o integral (Cano, 2013; Carratalá, 2012; Díaz et al, 2012; Horngren et al, 2012b).

Según Burbano (2010), Cano (2013) y Díaz et al (2012) y Donoso y Donoso (2017), el presupuesto de producción implica necesariamente que la empresa haya analizado su capacidad de producción, ya que de esta depende la realización y el cumplimiento de las metas. Para cumplirlas, la empresa debe contar con un plan de producción que le permita planear, coordinar y controlar todo lo relacionado con la fábrica (políticas en cuanto a tiempo de entregas, niveles óptimos de inventarios, almacenamiento, determinación de la capacidad instalada), las unidades que se van a producir y que se requieren para atender oportunamente la demanda, el proceso de producción y la calidad y cantidad de materias primas, mano de obra y costos indirectos a utilizar en el proceso. 
En ese mismo orden, el análisis del punto de equilibrio, el costo-volumenutilidad, el costeo directo y el absorbente, permitirán conocer los límites a utilizar para mantener el control de las ganancias o para evitar la generación de pérdidas. Éstos se analizan a través de ecuaciones, gráficos y la evaluación del estado de ingresos (Carratalá, 2012; Coyle et al, 2013; Hernández, 2012, 2014; Horngren et al, 2012a, 2012b; Jiménez, 2010; Lazo, 2013b).

Otro punto importante que permitirá un buen análisis de costos es la estandarización, la cual consiste en fijar de forma unitaria un costo o cantidad a utilizar de cada recurso que compone el costo del bien o servicio que se adquiere o produce. Los estándares se calculan en base a eficiencia (en cantidad y costo) y sirven de base para la elaboración de presupuestos. (Calleja, 2013; Hernández, 2012, 2014).

En el análisis de la eficiencia Hernández (2012), introduce el tema sobre el despilfarro en la producción y en el uso de los recursos, además del mal manejo de los equipos y el espacio físico de la planta.

Los costos de producción son el soporte fundamental para la fijación de precios, la cual puede realizarse considerando alguno de los siguientes métodos: costo total, costeo directo, rendimiento deseado, cláusulas escalatorias, valor económico agregado, competencia, valor percibido por el cliente o precios de transferencia (Calleja, 2013, Horngren et al, 2012b).

El precio de venta, al cual se ofrece el producto al público, debe responder a las necesidades de rentabilidad esperadas, ya que dependiendo de la fórmula que se utilice se puede recuperar solo el costo al adicionarle al mismo un porcentaje de utilidad determinado; o se puede recuperar, además del costo, los gastos operacionales; o más bien, como ocurre en algunas empresas, donde se incorpora no solo los aspectos anteriores, sino también los costos financieros y el porcentaje de impuesto a la renta (Díaz et al., 2012).

Es oportuno aclarar con respecto a los costos y los ingresos en los hoteles, que el precio de venta de un servicio suele fijarse sobre la base del análisis del precio de mercado; es decir, este precio depende de los precios de la competencia, de la categoría del hotel y de la política de marketing que se diseñe (Gómez, 2013).

\section{La toma de decisiones}

Después que se ejecutan, registran, resumen y analizan los costos, se procede a la toma de decisiones. Esta permitirá saber si se reducen los costos, implementan 


\section{Contexto caberas..}

nuevas estrategias de consumo, amplían, reducen o eliminan departamentos o líneas de productos, fijar precios de venta y qué hacer con los desechos sólidos generados y el impacto producido en la sociedad y el medioambiente.

La toma de decisiones, en cuanto a costo se refiere, se realiza en cinco pasos: 1) la obtención de la información; 2) la realización de predicciones acerca de costos futuros; 3) elección de una alternativa; 4) implementación de la decisión y 5) evaluación del desempeño (Ramírez, 2013). Estas decisiones se toman en cuenta analizando certidumbres, riesgos e incertidumbres (García, 2014; Lazo, 2013a), pues hasta el hecho de carecer de inventarios representa altos riesgos para las empresas, que afectan los costos, la productividad y la rentabilidad.

Otras decisiones que se toman en cuenta se refieren a la evaluación de la calidad, en productos y servicios, establecimiento de programas Justo a Tiempo, contratos laborales, comerciales y de construcción de obras, beneficios, inversiones, etc. (Carratalá, 2012).

Para tomar decisiones sobre reducción de costos, Carratalá expone diferentes técnicas que deben ser consideradas por los gerentes y contadores. Estas son: estudiar los procesos productivos; recurrir a la tercerización cuando sea necesario; mantener una buena relación con los proveedores; permitir la participación del personal en los procesos de planificación; invertir en calidad; mantener un flujo eficiente de las informaciones en la empresa; cuidar los aspectos logísticos y considerar la automatización de los procesos.

\section{Conclusiones}

Como ya se analizó, los costos de producción de bienes y servicios son de suma importancia para el estudio de la contabilidad y la administración de empresas. Estudiar contabilidad de costos permite a los contadores y administradores evaluar los recursos a utilizar, cuantificarlos y controlarlos para con ellos tomar decisiones que lograrán mantener a la empresa siendo competitiva en el mercado, frente a sus clientes y sus competidores.

Independientemente de los temas generales sobre costos, los autores citados tienen perspectivas diferentes que permiten ampliar los conocimientos necesarios para su adecuado control en cualquier ámbito. Por ejemplo, autores como Carratalá, Concepción, Horngren et al., Ramírez, Uribe, hacen mayor énfasis en la gestión administrativa de los costos; llevando con ello a conocer sobre cómo analizar las utilidades en relación a los costos y el volumen de producción; analizar flujos de efectivo y estados financieros, con la finalidad de medir rentabilidad económica, solvencia y utilidad. 
Cabrera y Mariano, Calleja, Hernández, Jiménez, Lazo y Rincón y Villareal, tienen mayor enfoque contable. En cambio, Abanto y Luján y Actualidad Empresarial, tienen un enfoque impositivo; Suárez en construcción, Gómez, y algunos ya mencionados, en producción de servicios. Burbano, Cano, en parte gestión, en parte contabilidad, se concentran más en los presupuestos.

Aunque no fueron incluidos en el contenido, existen otras referencias bibliográficas de actualidad que aportan conocimientos prácticos sobre el tema, que pueden ser de mucho provecho para el estudio y comprensión de la contabilidad de costos. Tal es el caso de Paredes (2017), Puruncajas (2010) y Ayuso y Barranchina (2011), quienes se enfocan en la aplicación de las NICs relacionadas a los inventarios (NIC 2) y a los resultados (NICs 11 y 23); así también como Aguirre (2013), quien hace referencia de los costos de productos y servicios con un enfoque de emprendimiento.

Otros autores, como Cuevas (2012), Del Río (2012), Escalante y Uribe (2017), Isidro (2014), Malloy Jiménez (2014), Neuner (2011a, 2011b), Rincón y Narváez (2017) y Rincón y Villarreal (2014b), aunque proponen temas interesantes en sus publicaciones, que realizan aportes significativos a la contabilidad de costos, no fueron consultados por no estar disponibles, al momento de la investigación, en las fuentes y bases de datos consultadas.

Con esto, se demuestra que se puede estudiar contabilidad de costos con recursos actualizados; la gran mayoría de ellos, con estilos de redacción de fácil entendimiento y de buena calidad.

\section{Referencia}

Abanto, M. y Luján, L. (2013). El gasto, el costo y el costo computable, análisis contable y tributario. Lima, Perú: Contadores y Empresas.

Actualidad Empresarial. (2014). Registro de costos, información mínima, formatos y casuísticas. Pacífico.

Aguirre, J. (2013). Emprendeduría práctica para hacer negocios. Ciudad de México, México. Alfaomega.

Ayuso, A. y Barranchina, M. (2011). Casos prácticos resueltos de contabilidad de costes. Profit.

Burbano, J. (2010). Presupuestos, un enfoque de direccionamiento estratégico, gestión y control de recursos. McGraw-Hill. 


\section{Contexto cabora, $M$}

Cabrera, M. y Mariano, D. (2015). Diagnóstico y optimización de la metodología de asignación de costos conjuntos en el punto de separación, caso: Comercial La Gran Parada, S.R.L. (Tesis de maestría inédita). Santo Domingo, República Dominicana. Universidad Nacional Pedro Henríquez Ureña.

Calleja, F. (2012). Costos. (2 $2^{\mathrm{a}}$ ed.). Ciudad de México, México: Pearson.

Cano, A. (2013) Contabilidad gerencial y presupuestaria, aplicada a las ciencias económicas, administrativas y contables. Ediciones de la $\mathrm{U}$.

Carratalá, J. y Albano, H. (2012). Gerenciamiento estratégico de costos, herramientas prácticas para los procesos de reducción de costos. Ciudad de México, México: Alfaomega.

Concepción, D. (2016). Contabilidad de gestión. Pirámide.

Coyle, Langley, Novack y Gibson. (2013). Adminsitración de la cadena de suminsitro. $\left(9^{\mathrm{a}} \mathrm{ed}\right)$. Cengage.

Cuevas, F. (2012). Control de costos y gastos en restaurantes. Limusa.

David, P. (2016). Logística internacional, administración de operaciones de comercio internacional. Cengage.

Del Río, C. (2012). Manual de costos, presupuestos y adquisiciones, y abastecimientos. Cengage.

Díaz, M., Parra, R. y López, L. (2012). Presupuestos, enfoque para la planeación financiera. Bogotá, Colombia: Pearson.

Díaz, J. (2010). Costos industriales sin contabilidad. Ciudad de México, México: Pearson.

Donoso, R. y Donoso, A. (2011) Sistemas de costeo e información económica. Madrid, España, Pirámide.

Escalante, J.y Uribe, R. (2017). Variables críticas en la gestión de costos. Bogotá, Colombia: Alfaomega.

García, J. (2014). Contabilidad de costos. (4ª ed). México: McGraw-Hill.

Gómez, A. (2013). Cómo estructurar un sistema de costos en una empresa de servicios, guía operativa del contador. Contadores y empresa. Recuperado de https://www.coursehero.com/file/30203981/COMO-ESTRUCTURAR-UN-SISTEMA-DE-COSTOS-EN-UNA-EMPRESA-DE-SERVICIOSpdf/ 
Heredia, L. (2013). Gerencia de compras, la nueva estrategia competitiva. (2 ed.). Bogotá, Colombia: Ecoe Ediciones.

Hernández, N. (2012). Contabilidad de costos de productos y servicios, una herramienta indispensable en la gestión competitiva de los negocios. Santo Domingo: Búho, S.R.L.

Hernández, N. (2014). Contabilidad de costos de productos y servicios, una herramienta indispensable en la gestión competitiva de los negocios. $\left(2^{\mathrm{a}}\right.$ ed.). Santo Domingo: Búho, S.R.L.

Horngren, C., Datar, M. y Foster, G. (2012a). Contabilidad de costos, un enfoque gerencial. (12 ${ }^{\mathrm{a}}$ ed.). México: Pearson.

Horngren, T., Datar, M. y Rajan, M. (2012b). Contabilidad de costos, un enfoque gerencial (14 ed.). México: Pearson.

Instituto Mexicano de Contadores Públicos (2016). NIIF (Parte A). México.

Isidro, G. (2014). Contabilidad de costos para la toma de decisiones, aplicación práctica. Perú: Instituto Pacífico.

Jiménez, W. (2010). Contabilidad de costos. Bogotá, Colombia: Fundación para la educación superior San Mateo.

Lazo, M. (2013a). Contabilidad de los costos I. Programa de educación superior a distancia - Proesad: Perú.

Lazo, M. (2013b). Contabilidad de los costos II. Programa de educación superior a distancia - Proesad: Perú.

Mallo, C. y Jiménez, M. (2014). Contabilidad de costes. Pirámide.

Pabón, H. (2012). Fundamentos de costos. México: Alfaomega.

Paredes, J. (2017). Casos prácticos de gestión de costes. Pirámide.

Puruncajas, M. (2010). NIIF Normas internacionales de información financiera, casos prácticos. Ecuador: Universidad Técnica Particular de Loja.

Ramírez P., D.N. (2013). Contabilidad administrativa, un enfoque estratégico para competir. ( $9^{\text {a }}$ ed.). McGraw-Hill.

Rincón y Narváez (2017). Presupuestos bajo NIIF. Ediciones de la U. 


\section{Contexto caboras,,}

Rincón y Villarreal (2014a). Costos I, con aproximaciones a las NIC 02 y NIIF 08. Ediciones de la U.

Rincón y Villarreal (2014b). Costos II, con aproximaciones a las NIC 02 y NIIF 08. Ediciones de la U.

Suárez S., C. (2017). Costo y tiempo en edificación. ( $3^{\mathrm{a}}$ ed.). México: Limusa.

Tafur, J.C. \& Osorio, J. A. (2013). Costeo basado en actividades ABC: gestión basada en actividades ABM. Colombia: Ecoe Ediciones.

Tafur, J.C. \& Osorio, J. A. (2016). Costeo basado en actividades ABC: gestión basada en actividades $A B M$. ( $2^{\mathrm{a}}$ ed). Colombia: Ecoe Ediciones.

Uribe M, R. (2011). Costos para la toma de decisiones. Colombia: McGraw-Hill.

Vanderbeck, E. y Mitchell, M.R. (2017) Principios de contabilidad de costos. (17 ed.). Cengage.

Warren, C. S. (2010). Contabilidad administrativa (10a ed). (L. Campa Rojas, Trad.) México: Cengage.

\section{Para citar este artículo:}

Cabrera, M. (2017). La contabilidad de costos en la producción de bienes y servicios. Revisión bibliográfica actualizada (2010-2018). EnContexto, 6(9), 203-230. 